\title{
To improve the quality of life in elderly people with fragility fractures
}

\author{
PY Lau, FHKCOS, FHKAM (Orthopaedic Surgery) \\ Department of Orthopaedics and Traumatology, United Christian Hospital, Hong Kong
}

Hong Kong Med J 2016;22:4-5

DOI: 10.12809/hkmj154782

The global increasing elderly population is placing a great burden on the financial and health systems of all countries. A major source of this burden is fragility fracture. ${ }^{1}$ In Hong Kong, around 6000 patients present each year with hip fracture, and current projections indicate that the numbers will double by $2050.2,3$

Fragility fracture has long been a major source of the workload for orthopaedic departments in Hong Kong. Patients with hip, vertebral, or wrist fracture occupy a high percentage of orthopaedic beds in all public hospitals. Most hip fractures require either internal fixation or hip replacement to alleviate fracture pain and allow early ambulation. ${ }^{4,5}$ A decade ago, most hip fracture patients in Hong Kong would wait 5 to 6 days for surgery because health personnel-including orthopaedic surgeons, anaesthetists, and nurses-did not consider the condition to be important. Nonetheless such a delay is now known to increase in-patient and postoperative mortality and morbidity. The Blue Book of the British Orthopaedic Association 2007 stated that hip fractures should be operated on within 48 hours. ${ }^{2}$ In 2009, the Hospital Authority selected geriatric hip fracture as the first Key Performance Indicator for orthopaedics in Hong Kong. ${ }^{6}$ The aim was to confine preoperative stay to no more than 2 days for $70 \%$ of hip fracture patients. Prior to 2007 this figure was approximately $30 \%$, but had improved to $62 \%$ by August 2008. The mean preoperative length of stay has now been reduced by 3.5 days, from the previous 6 days. By 2009, the hard work of all orthopaedic surgeons, geriatricians, and allied health colleagues had shortened the waiting time to 2 days in $70 \%$ of patients. ${ }^{6}$ Postoperative mortality and morbidity are also much reduced and, more importantly, the length of time the patient has fracture pain. These elderly now walk earlier and are discharged earlier. Thus their quality of life is improved and more hospital beds are available for other patients.

There are a few tests that help the orthopaedic surgeons to assess mortality risks of hip fracture patients. They are discussed in one of the articles in this issue, and improve communication between the doctor and patient's family, as well as minimising any misunderstanding. ${ }^{7}$

In patients with vertebral fracture, treatment is mostly conservative although some suffer significant back pain and may be bedridden for a few months. Nonetheless with advances in technology patients who do not respond well to conservative treatment now benefit from vertebroplasty, which implies injection of bone cement into the fractured vertebra. ${ }^{8}$ Good pain relief is achieved in many patients postoperatively, enabling early rehabilitation.

Wrist fracture is a very common problem in the elderly after a fall. For a long time, treatment was focused on closed reduction and application of plaster-of-Paris (POP) although such plaster immobilisation resulted in stiffness and pressure sores. Patients often required a long period of physiotherapy to regain movement. Internal fixation was seldom performed because the failure rate with old implants was high. The development of new locking anatomical plates and bone substitute has greatly improved the success rate of surgery and these patients can now move their wrist much earlier following surgery and avoid the complications associated with POP immobilisation.

With increasing use of new surgical techniques and implants, the number of operative complications is expected to rise. The price of the implants is also considerably increased and management of complications is often difficult in these elderly patients. This may place increasing demands on hospital services and budget. Adequate training and supervision of junior doctors is required to ensure the job is done well.

Prevention is always better than treatment. Several osteoporotic drugs are widely used to help in the treatment and prevention of osteoporosis. Their use is usually long-term and they are not cheap, however.

Apart from osteoporosis, sarcopenia is another factor that causes fall of the elderly and is also discussed in this issue of the journal. ${ }^{9}$ Paying more attention to nutrition is very important in these elderly to build up muscle bulk. Many of these patients have medical co-morbidities so collaboration of geriatricians with orthopaedic surgeons is of utmost importance to ensure uninterrupted and well-coordinated pre- and post-operative care. All patients with fragility fracture after a fall should be offered a multidisciplinary service to prevent another fall. It is advisable for public hospitals to organise a team of staff that includes orthopaedic 
surgeons, geriatricians, allied health colleagues and nurses with special interest in this field to manage these patients with fragility fracture together.

Longevity is nothing to admire, rather we should pursue a better quality of life for our senior citizens. Looking after patients with fragility fracture well is a lot cheaper than looking after them badly. The Hong Kong SAR Government and community should invest more in the care of these patients. The rewards can be surprisingly high.

\section{References}

1. American Academy of Orthopaedic Surgeons. Position statement: Recommendations for enhancing the care of patients with fragility fractures. June 2003. Revised December 2009. Available from: http://www.aaos.org/ CustomTemplates $/$ Content.aspx id $=22324 \&$ ssopc $=1$. Accessed Dec 2015.

2. The care of patients with fragility fracture. British Orthopaedic Association. September 2007. Available from: http://www.fractures.com/pdf/BOA-BGS-Blue-Book.pdf. Accessed Dec 2015.

3. Man LP, Ho AW, Wong SH. Excess mortality for operated geriatric hip fracture in Hong Kong. Hong Kong Med J
2016;22:6-10.

4. Hip fracture: management. Clinical guideline. National Institute for Health and Care Excellence (NICE). 22 June 2011. Available from: http://www. nice.org.uk/guidance/cg124/resources/hip-fracturemanagement-35109449902789. Accessed Dec 2015.

5. Chan VW, Chan PK, Chiu KY, Yan CH, Ng FY. Why do Hong Kong patients need total hip arthroplasty? An analysis of 512 hips from 1998 to 2010. Hong Kong Med J 2016;22:11-5.

6. Report of the Chairman. COC in Orthopaedics and Traumatology. Hong Kong: Hospital Authority; 2009.

7. Lau TW, Fang C, Leung F. Assessment of postoperative short-term and long-term mortality risk in Chinese geriatric patients for hip fracture using the Charlson comorbidity score. Hong Kong Med J 2016;22:16-22.

8. Heini PF, Wälchli B, Berlemann U. Percutaneous transpedicular vertebroplasty with PMMA: operative technique and early results. A prospective study for the treatment of osteoporotic compression fractures. Eur Spine J 2000;9:445-50.

9. Ho AW, Lee MM, Chan EW, et al. Prevalence of presarcopenia and sarcopenia in Hong Kong Chinese geriatric patients with hip fracture and its correlation with different factors. Hong Kong Med J 2016;22:23-9. 